\title{
LEGADO, CRÍTICA Y SUPERACIÓN DEL IDEARIO DE LA REFORMA UNIVERSITARIA DE 1918: JULIO V. GONZÁLEZ Y LA RADICALIZACIÓN DEL PENSAMIENTO REFORMISTA
}

\author{
DOI: http://dx.doi.org/10.1590/2236-3459/77890 \\ Claudio Suasnábar \\ Universidad Nacional de La Plata (UNLP), La Plata/Buenos Aires, Argentina
}

$\cos 80$

\begin{abstract}
Resumen
El artículo analiza el pensamiento universitario de Julio V. González (ex dirigente estudiantil y primer historiador de la Reforma) como expresión, por un lado, de las disputas por definir el imaginario reformista como legado, crítica y superación de este corpus de ideas, y por otro, de las tensiones entre intelectuales y partidos de izquierda en la Argentina que se manifiestan en un cambio en las modalidades de intervención intelectual y posicionamiento político. El trabajo está organizado en dos secciones: la primera explora la caracterización de la Reforma Universitaria de González y su ubicación en el contexto socio-político. La segunda sección analiza la reflexión posterior de González y su propuesta de emancipación de la universidad que marca una radicalización del pensamiento reformista.

Palabras clave: Reforma Universitaria de 1918, universidad, intelectuales, política, Argentina.
\end{abstract}

\section{LEGADO, CRÍTICA E SUPERAÇÃO DA IDEOLOGIA DA REFORMA UNIVERSITÁRIA DE 1918: JULIO V. GONZÁLEZ E A RADICALIZAÇÃO DO PENSAMENTO REFORMISTA}

\begin{abstract}
Resumo
O artigo analisa o pensamento da universidade de Julio V. González (ex-líder estudantil e primeiro historiador da Reforma) como expressão, por um lado, das disputas para definir o imaginário reformista como legado, crítica e superação deste corpus de idéias e por outro lado, das tensões entre intelectuais e partidos de esquerda na Argentina que se manifestam em uma mudança nas modalidades de intervenção intelectual e posicionamento político. O trabalho está organizado em duas seções: a primeira explora a caracterização da Reforma Universitária de González e sua localização no contexto sociopolítico. A segunda seção analisa a reflexão posterior de González e sua proposta de emancipação da universidade que marca uma radicalização do pensamento reformista.
\end{abstract}

Palavras-chave: Reforma Universitária de 1918, universidade, intelectuais, política, Argentina.

\section{LEGACY, CRITICISM AND OVERCOMING THE IDEOLOGY OF THE UNIVERSITY REFORM OF 1918: JULIO V. GONZÁLEZ AND THE RADICALIZATION OF REFORMIST THOUGHT}

\section{Abstract}

The article analyzes the university thought of Julio V. González (former student leader and first historian of the Reformation) as an expression, on the one hand, of the disputes to define the reformist imaginary as legacy, criticism and overcoming of this corpus of ideas, and on the other hand, of the tensions between intellectuals and leftist parties in Argentina that manifest themselves in a change in the modalities of intellectual intervention 
and political positioning. The work is organized into two sections: the first explores the characterization of González's University Reform and its location in the socio-political context. The second section analyzes González's subsequent reflection and his emancipation proposal from the university that marks a radicalization of reformist thought.

Keywords: University Reform of 1918, university, intellectuals, politics, Argentina.

\section{HÉRITAGE, CRITIQUE ET DÉPASSEMENT DE L'IDÉOLOGIE DE LA RÉFORME UNIVERSITAIRE DE 1918: JULIO V. GONZÁLEZ ET LA RADICALISATION DE LA PENSÉE RÉFORMISTE}

\section{Résumé}

L'article analyse la pensée universitaire de Julio V. González (ancien dirigeant étudiant et premier historien de la Réforme) comme l'expression, d'une part, des disputes pour définir l'imaginaire réformiste comme héritage, critique et dépassement de ce corpus d'idées, et d'autre part, des tensions entre intellectuels et partis de gauche en Argentine qui se manifestent par un changement dans les modalités d'intervention intellectuelle et de positionnement politique. Le travail est organisé en deux sections: la première explore la caractérisation de la réforme universitaire de González et sa localisation dans le contexte sociopolitique. La deuxième section analyse la réflexion ultérieure de González et sa proposition d'émancipation de l'université qui marque une radicalisation de la pensée réformiste.

Mots-clés: Réforme universitaire de 1918, université, intellectuels, politique, Argentine. 


\section{Introducción}

E. n unos pocos meses se cumplirán 100 años de la Reforma Universitaria de Córdoba cuyo impacto y proyección continental dejó una huella indeleble no solo en la configuración histórica de nuestras instituciones sino más importante aún, en el imaginario político-intelectual de los actores académicos. El "Grito de Córdoba" como se conocerá la revuelta estudiantil comienza con aquel llamado a "los hombres libres de Sudamérica" para denunciar el anacronismo del régimen universitario y anunciar el inicio de una nueva época: "Creemos no equivocarnos, las resonancias del corazón nos lo advierten: estamos pisando sobre una revolución, estamos viviendo una hora americana"1.

El Manifiesto Liminar como texto pertenece claramente al género de las proclamas o declaraciones que caracterizan la emergencia de las vanguardias estético-culturales y políticas de fines del siglo XIX y principios del siglo XX. El futurismo, el surrealismo y el dadaísmo tuvieron sus propios manifiestos, que se suman al Manifiesto Comunista de Marx y Engels y al J'accuse (Yo acuso) de Emile Zola que expresará a los intelectuales franceses, todas expresiones que marcan el nacimiento de estos movimientos y corrientes de pensamiento. Como acta de fundación, los manifiestos combinan, por un lado, una dimensión programática que define el posicionamiento de ruptura del grupo políticointelectual frente a otros segmentos dentro su esfera o campo específico y, por otro lado, delimitan un imaginario discursivo que por definición desborda la propia dimensión propositiva y se proyecta como un horizonte utópico.

En el caso del Manifiesto Liminar de la Reforma de Córdoba esta primera dimensión se expresa en el demos universitario que articula la noción de co-gobierno como principio de representación (derecho a participar de los estudiantes en paridad de condiciones con los profesores), y la noción de autonomía como principio de soberanía, autoconsciencia y capacidad de la institución universitaria. La democracia universitaria se complementa y profundiza con la reforma pedagógica que pone en el centro al estudiante y su rol activo en el aprendizaje y formación, y desplaza la cátedra vitalicia por libre asistencia a clase y la libertad de cátedra, así como también la vinculación entre enseñanza teórica y la práctica. Desbordando este programa de cambio universitario, el Manifiesto Limitar demarca un imaginario discurso de regeneración social cuyos rasgos principales articularán distintos pares conceptuales como reforma universitaria y revolución social, crisis del antiguo régimen y nueva civilización, latinoamericanismo y antimperialismo, juvenilismo y vieja generación, entre otros.

Como señala Carlos Tunnerman Bernheim (2008), el movimiento reformista fue más exitoso en la democratización del gobierno universitario que con distintos grados de representación y participación incluyeron a los estudiantes, logro que fue acompañado por reformas pedagógicas en las formas de enseñanza en casi todas las instituciones de la región. (MUNERA RUíZ, 2011). Así, la concreción de buena parte de la dimensión programática de la Reforma se volverá constitutiva de la identidad de la universidad latinoamericana, la cual por derecho propio reclamará ser reconocida como otro modelo histórico junto con la universidad humboltiana y napoleónica. Por el contrario, el imaginario

${ }^{1}$ La juventud argentina de Córdoba a los hombres libres de Sudamérica: Manifiesto Liminar de 21 de junio de 1918.

\begin{tabular}{|l|l|l|l|l|l|}
\hline Hist. Educ. (Online) & Porto Alegre & v. 22 & n. 54 & jan./abr. 2018 & p. 174-189
\end{tabular}


reformista desde el mismo origen del movimiento será motivo de controversia entre diferentes tradiciones políticas que disputaran no solo por construir una narrativa histórica de la Reforma y una explicación de sus causas sino principalmente confrontaran para definir el sentido y orientación de aquel horizonte utópico.

No es casual, entonces que la proyección latinoamericana del ideario reformista haya adoptado diferentes configuraciones ideológicas según los países y tradiciones políticas que reapropiaron y resignificaron aquel corpus de ideas. Así, en la década de 1920 el peruano José Carlos Mariátegui y el cubano Julio Antonio Mella inscribirán el legado de la reforma en la continuidad del pensamiento marxista, mientras que Víctor Raúl Haya de la Torre también peruano encontrará en esas mismas ideas, las razones para conformar un nuevo movimiento político, la Alianza Popular Revolucionaria Americana (Apra) de carácter nacional-popular. Frente a estas dos posiciones políticas que, ciertamente, tomarán caminos diferentes en las décadas posteriores, existirá una tercera alternativa que, como bien señala Néstor Kohan (2000), intentará proyectar la experiencia de la lucha estudiantil hacia el terreno político.

La figura de Julio V. González es quién mejor representa el derrotero intelectual y político de un segmento de la dirigencia estudiantil que visualizará en la Reforma Universitaria no solo la expresión del cuestionamiento a la universidad tradicional sino más importante aún, el surgimiento de una nueva generación cuya misión fundamental sería la de realizar una transformación social profunda². Este movimiento de pasaje entre una visión de la reforma como expresión del descontento estudiantil y la autoconciencia como nueva generación, finalmente, encontrará en la tradición del "socialismo humanista", una alternativa diferente a la ortodoxia marxista y el nacionalismo popular. Ciertamente, esta radicalización del pensamiento reformista expresa el cambio en las modalidades de intervención del "intelectual comprometido" al "intelectual orgánico" que se incorporará al Partido Socialista pero que - como veremos más adelante - conservará una mirada autónoma y crítica aún de las posiciones partidarias.

Julio V. González (1899-1955) participó activamente en la Reforma Universitaria de Córdoba, ciudad donde estuvo en julio de aquel año como representante de la Federación Universitaria de La Plata, siendo elegido secretario del Primer Congreso Nacional de Estudiantes que se reunió en dicha ciudad. Entre 1919-1920, fue el presidente más joven de la FUA (Federación Universitaria Argentina) y luego consejero estudiantil, entre muchas otras tareas que desempeñó. Más tarde, también será miembro titular del consejo directivo de la Unión Latinoamericana y codirector de Sagitario, Revista de Humanidades. En 1929, llegó a ser elegido decano "revolucionario" en una toma de la Facultad de Derecho de Buenos Aires. Hijo de Joaquín V. González, fundador de la Universidad de La Plata y miembro prominente de la elite política e intelectual de 1880, se afiliará al Partido Socialista a comienzos de los años 1930, siendo electo diputado nacional por dicho partido en 1941.

Esta experiencia en la lucha estudiantil marcó su formación política e ideológica

\footnotetext{
2 "La Reforma Universitaria comenzó por ser una revuelta de estudiantes, sirvió luego para la definición ideológica de una nueva generación en América Latina, y ha terminado en el mito de los hombres jóvenes del continente sud. En 1918 un reformista era el estudiante universitario sublevado contra sus maestros, en 1921 el americano de la Nueva Generación que declaraba su divorcio con el pasado y su disconformidad con el estado de cosas y sistema de ideas que se regía la comunidad de América, y en 1925 un hombre entregado a un ideal reconstructivo tocado de un fuerte sentido socialista." In: GONZÁLEZ, Julio V. La Reforma Universitaria. Buenos Aires: Ediciones de la Revista Sagitario, 1927, p. 12.
}

Hist. Educ. (Online)

Porto Alegre

v. 22

n. 54

jan./abr. 2018

p. $174-189$ 
que se manifestará una vasta producción intelectual constituyéndose en uno de los principales creadores del ideario reformista. En este sentido, esta comunicación tiene por objetivo analizar el pensamiento universitario de Julio V. González como expresión, por un lado, de las disputas por definir el imaginario reformista como legado, crítica y superación de dicho corpus de ideas, y por otro, de las tensiones entre intelectuales y partidos de izquierda en la Argentina que, como señalamos, está estrechamente ligado al cambio en las modalidades de intervención intelectual y posicionamiento político. Planteado de esta manera, el trabajo está organizado en dos secciones: la primera explora la caracterización de la Reforma Universitaria de González y su ubicación en el contexto socio-político. La segunda sección analiza la reflexión posterior de González y su propuesta de emancipación de la universidad que marca una radicalización del pensamiento reformista. Hacia el final y a modo de conclusión se presenta algunos comentarios sobre la vigencia del pensamiento de González en el debate actual de la universidad argentina.

\section{La reforma universitaria como movimiento social y político}

Apenas cinco años trascurridos de la revuelta estudiantil en Córdoba, Julio V. González comenzaría una reflexión y análisis de la Reforma Universitaria en la cual visualiza no solo un problema restringido a las aulas sino la expresión de la crisis de civilización occidental, y a la vez, la llegada de una nueva sensibilidad que augura un cambio profundo en las sociedades contemporáneas. De ahí que la ubicación histórica y significado de la Reforma deba pensarse como parte de la "cuestión social"3 que emerge con fuerza en las primeras décadas del siglo XX como resultado de la convergencia de tres procesos: la guerra europea, la revolución rusa y el advenimiento del radicalismo al poder en la Argentina.

La primera guerra mundial (1914-1918), con su saldo de muerte de más de diez millones de personas y una Europa desbastada, expresa precisamente la crisis de la civilización occidental. En la mirada de González, el conflicto bélico representaba la bancarrota de los ideales y promesas de progreso que la sociedad occidental había puesto en el libre juego de fuerzas y que ahora ser revelaba como destrucción y caos. Pero si la guerra aparece como el fin de una época, la aparición de la Revolución Rusa en el escenario internacional constituye "el símbolo de un idealismo rebelde y reconstructor" que augura la llegada de un tiempo nuevo que trae consigo "ideales de humana redención". Lejos de una lectura economicista como el que caracterizará a buena parte de los partidos comunistas, González destaca el contenido espiritual de la revolución rusa en la medida que recupera "aquellos valores filosóficos y morales que la humanidad ha perdido con la guerra mundial". (GONZÁLEZ, 1927, p. 97-98).

En rigor, esta mirada ético-político y culturalista de la revolución rusa fue introducida por José Ingenieros, quién será considerado "maestro de juventud" e intelectuales faro de toda la generación de estudiantes reformistas como González. Desde esta perspectiva, la crisis de la civilización occidental presenta dos caras: por un lado, la guerra redentora de

3 "Digámoslo claramente, entonces: la Reforma Universitaria es parte de una cuestión social, que el desarrollo material y moral de nuestra sociedad ha impuesto a raíz de la crisis producida por la guerra." (p. 109). En GONZÁLEZ, Julio V. Significación social de la Reforma Universitaria. In: Reforma Universitaria. 2 volúmenes. Buenos Aires: Edición de la Revista Sagitario, 1927. (Tomado de GONZÁLEZ, Julio V. La Universidad. Teoría y acción de La Reforma. Buenos Aires: Claridad, 1945, las citas siguientes corresponden a la misma edición). 
los pueblos que encarna la revolución rusa, promotora de nuevas valores e ideales, y por otro su reverso que es la guerra de los "bárbaros", como caracteriza a las naciones que impulsadas por un materialismo y consumismo ciego llevaron a la bancarrota de la civilización.

La llegada del radicalismo al poder constituye el tercer acontecimiento que marca el contexto de surgimiento de la Reforma Universitaria. En 1916 accedía a la presidencia de la Nación Hipólito Irigoyen, dando inicio a la primera experiencia de democracia política en la Argentina, y a la vez, dando por finalizada la dominación oligárquica de la elite política que desde 1880 había dirigido los destinos del país. Como muchos intelectuales, González visualiza el carácter contradictorio del radicalismo que, si bien representa una nueva fuerza popular, por otro lado, en su discurso y práctica resulta continuadora de las tradiciones caudillescas y atrasadas de la cultura política nacional. ¿Entonces, que traía de nuevo el nuevo el radicalismo? "Concretamente nada" responde, pero, no obstante - señala González - es una fuerza política en cierto modo "creadora y fecunda" que arrasaba, pero "dejando el limo fértil de la sensibilidad netamente popular llegar a las esferas de gobierno". (GONZÁLEZ, 1945, p. 112).

Así, la Reforma Universitaria surgida en el contexto de la guerra europea, la revolución rusa y la llegada del radicalismo al gobierno no constituye un hecho aislado ni restringido a las aulas sino más bien se entronca en la crisis de civilización y en la emergencia de una época de realización de los ideales humanistas y de renovación social. Por ello, González no duda en señalar que:

[...] la Reforma Universitaria no fue el fruto de una concepción abstracta, ni el triunfo de una escuela filosófica, ni la imposición de un grupo de mentalidades privilegiadas, fue la explosión de un estado de conciencia social que se había formado alrededor de los cristalizados centros de cultura. (GONZÁLEZ, 1945, p. 114).

En su visión, las universidades representaban claramente ese estado de conciencia social en la medida que ellas (y particularmente la de Córdoba) era el reducto aristocrático y matriz de formación de los privilegios de la clase dominante. En ese sentido, y como señalamos en otros trabajos, los principios que introduce el movimiento reformista adquieren su significación en la particular coyuntura que caracteriza este primer experimento democrático y el ascenso de nuevos sectores sociales. Así, la idea del cogobierno recupera metafóricamente el modelo de la república democrática donde el "pueblo" está representado por los claustros, mientras que los consejos superiores y académicos adquieren simultáneamente la forma de "parlamentos", y de "gobierno" universitario. (SUASNÁBAR, 2009). Como bien da cuenta la cita siguiente, el "demos" universitario reclamado por los estudiantes rápidamente se resignficaría en una demanda de democratización social. Así, señala que:

La renovación universitaria fue bien pronto renovación social, el repudio revolucionario de los dogmas de orden y autoridad, proclamado dentro de la casa de estudios, lo fue igualmente y en el mismo tono frente a los que sojuzgaban en las sociedad, la condenación del obscurantismo religioso que ahogaba la libertad de conciencia desde la cátedra, se repitió ampliando su eco contra el mismo que asfixia desde el púlpito y desde el seno de la clase aristocrática, el desprecio hacia los maestros del aula, se trocó en el escenario nacional, en un hondo e irreconciliable divorcio entre la nueva y la vieja generación, el nuevo sentido de la 
democracia, creado sobre nuevos conceptos de disciplina, jerarquía y voluntad popular, enunciados como base de la llamada democracia universitaria, fue lema de combate en el ambiente social, evidenciados en forma de una interpretación propia de las fuerzas vivas de la comunidad. (GONZÁLEZ, 1945, p. 117-118).

Pero la Reforma Universitaria no solo era expresión de esta conciencia social renovadora sino también traía consigo al actor social: la "Nueva Generación" que cumpliría la misión de refundar las bases morales y sociales de aquella civilización en crisis. A diferencia de la tradición marxista que encuentra en el proletariado al actor social sepulturero de la sociedad capitalista, González toma del filósofo español José Ortega y Gasset su teoría de las generaciones, la cual explica el movimiento histórico como una sucesión de generaciones ${ }^{4}$. ¿Qué es, entonces, una generación? "Por generación histórica - nos dice González - debe entenderse no la descendencia biológica de los hijos con respecto a los padres o los nietos hacia sus abuelos, sino la sucesión de hombres que en una época y lugar determinado aparecen y se distinguen por una sensibilidad e ideología propias, con las cuales realizan una labor conjunta que llena un periodo histórico". Así, generación y período histórico se entremezclan porque en el "generacionismo ortegiano" solo el período histórico se vuelve tal en la "obra cumplida por una generación". (GONZÁLEZ, 1945, p. 133).

Desde esta perspectiva, González reconstruye la historia argentina como la sucesión de cuatro generaciones: la "emancipadora" de 1910 conformada por los próceres de la revolución de Mayo y las luchas de independencia, la "precursora" de 1937 que pensarán el proyecto de nación en los que incluye a Echeverría, Alberdi y Gutiérrez, entre otros, la generación "constituyente" de 1853 que elaborará y fundará el Estatuto jurídico de la República, y la generación "organizadora" que si bien continúa el legado anterior a través de la creación de la superestructura institucional, se transformará en "una elite de caudillos ilustrados" que darán por finalizada la tarea de construcción del Estado Nacional. Para González, la "Nueva Generación" que representan los jóvenes reformistas tiene un fin "esencialmente liquidador y reconstructivo" porque no solo llega para declarar por concluida la tarea de la vieja generación sino también porque la nueva generación es la que "interpreta las necesidades, las aspiraciones y los sentimientos colectivos propios de una conciencia nacional en formación". (GONZÁLEZ, 1945, p. 139). Por ello la Reforma Universitaria expresa mucho más que un conflicto académico ya que como señalará en un texto posterior:

\begin{abstract}
El alzamiento de los estudiantes contra sus maestros era el duelo a muerte entre la nueva y la vieja generación, el enunciado de un hecho histórico llamado a llenar la época, y que consistía en el divorcio de dos generaciones. Ellos eran los restos de la del 80 cuyo ciclo habíase cerrado, nosotros representábamos la de 1918, que bajo la enseña de la Reforma Universitaria llegaba para reemplazarlos y juzgarlos. (GONZÁLEZ, 1931, p. 40). ${ }^{5}$
\end{abstract}

\footnotetext{
${ }^{4}$ Para un análisis pormenorizado de la noción de generación en Julio V. González y su trayectoria política véase: HERRERA, Carlos Miguel. Generación y política en Julio V. González. Revista Socialista, Buenos Aires, n. 1, Cuarta Época, Año I, junio 2009.

${ }^{5}$ En el mismo texto caracteriza de la siguiente manera la nueva generación: "Generación nihilista, iconoclasta, liquidadora, eran los primeros motes con que ensayábamos nuestra propia definición. Llegábamos para negarlo todo. No éramos nosotros quienes provocábamos el derrumbe. Empezamos caminando sobre escombros", en la p. 7.
} 
El término "nueva generación" será resignificado por la influencia del antiimperialismo latinoamericanista que el uruguayo José Enrique Rodó difundirá a través de su libro Ariel y que también será tematizado por el cubano José Martí y el nicaragüense Rubén Darío. No es casual, entonces, que en 1922 José Ingenieros y Aníbal Ponce convocaran a formar la "Unión Latinoamericana" como organismo coordinador de la acción de escritores e intelectuales del continente orientado a la difusión de la nueva conciencia antiimperialista. González y numerosos jóvenes universitarios participaran activamente en esta experiencia que ayudará a difundir y profundizar el sentido antiimperialista y latinoamericano del movimiento reformista.

Ciertamente, la nueva generación que encarnaba "el hombre nuevo que avanza bajo el emblema de la Reforma" había legitimado una modalidad de intervención política que posibilitaba a los intelectuales universitarios tomar posición frente a los problemas y debates nacionales e internacionales. Con todo, los años previos al golpe militar de 1930 signados por la parálisis del gobierno radical y el creciente autoritarismo, persuadirán a González de la necesidad de transformar el movimiento reformista en un partido político siguiendo el ejemplo del Apra de Haya de la Torre en Perú. En rigor, la creación de un Partido Nacional Reformista que propondrá en 1927 era el paso natural de un movimiento que desde sus inicios se planteó con un sentido político ${ }^{6}$. Así, en el marco de un acto organizado por la Federación Universitaria de Buenos Aires (Fuba) lanzará el llamado a la conformación del nuevo partido político:

\begin{abstract}
Pues bien, si al cerrar el primer ciclo, la Reforma Universitaria se manifiesta como un movimiento mediante el cual se ha formado dentro de la universidad una nueva generación histórica, munida de un ideario, ejercitada en las disciplinas de la acción y provista para la lucha de organismo y técnica propios, si la Reforma Universitaria es un fenómeno real, concreto, permanente y definitivamente incorporado a la vida de esta República, si la Reforma Universitaria es una organización mediante la cual los hombres nuevos que pasan por las universidades vienen, sin solución de continuidad, interviniendo en la discusión de los negocios públicos, si la Reforma Universitaria, en fin ha creado una nueva generación de políticos, yo os pregunto:¿Qué estáis esperando para proclamar a la faz del país la existencia del gran Partido Nacional Reformista? (GONZÁLEZ, 1968, p. 335).
\end{abstract}

La propuesta nunca llegaría a concretarse porque ciertamente la presencia por derecha del partido radical y del partido demócrata progresista, y por izquierda de socialistas y comunistas dejaba poco espacio para la formación de un nuevo partido que intentaba combinar elementos ideológicos de "reformismo social" y "socialismo humanista". Producido el golpe militar de 1930, González y un numeroso grupo de intelectuales universitarios (Carlos Sanchez Viamonte, Alejandro Korn, Deodoro Roca, entre otros) se incorporarán al Partido Socialista delineando un cambio en la modalidad de intervención ahora como "intelectuales de partido"7. No obstante, en grupo intelectual mantendrá una

6 "Hace diez años que estáis haciendo política, que a título de Reforma Universitaria os venís mezclando en
la discusión de los negocios públicos, no dejando pasar un solo acontecimiento sin abordarlo y pronunciaros
sobre él. Hace diez años que estáis elaborando un nuevo sistema de ideas dentro de la universidad,
sacadlas de una vez a la luz y sembrad en el surco que abráis como políticos la semilla acopiada como
estudiante reformista en el granero universitario. No dejéis que la ideología forjada con el esfuerzo de una
década se pierda en la abstracción". Véase en: GONZÁLEZ, Julio V. El Partido Nacional Reformista. In:
CIRIA, Alberto; SANGUINETTI, Horacio. Los reformistas. Buenos Aires: Jorge Alvárez, 1968. p. 334.
7 Sobre el derrotero político de González y este grupo intelectual, véase: GRACIANO, Osvaldo. Entre la torre
de marfil y el compromiso político. Intelectuales de izquierda en la Argentina. 1918-1955. Buenos Aires: 
autonomía relativa respecto de las orientaciones partidarias que se revela en los posicionamientos políticos e intelectuales de González no solo en la cuestión universitaria sino en las discusiones que al interior del partido generará la emergencia del peronismo en los años cuarenta y cincuenta ${ }^{8}$.

\section{La radicalización del pensamiento reformista: la Universidad Libre y la idea de "emancipación de la universidad"}

Hacia 1927 con la publicación de La Reforma Universitaria, González comienza un proceso de elaboración y sistematización de los principios que conformarán el corpus teórico del movimiento reformista. Cinco años antes había publicado La Revolución Universitaria 1918-1919 (1922), donde realiza la primera reconstrucción histórica de la lucha de los estudiantes de Córdoba, como también de otros dos acontecimientos que se inscriben en el clima de movilización en el ámbito educativo como son la huelga de maestros en Mendoza y la revuelta del Colegio Nacional de la ciudad de Chivilcoy.

Así, en los dos volúmenes que componen La Reforma Universitaria reúne una serie de textos (la mayoría producto de intervenciones públicas) en los cuales además de desarrollar lo que él denomina "el principio de injerencia estudiantil en el gobierno de la universidad", esto es, el co-gobierno, también profundiza en el resto de las demandas de renovación pedagógica impulsadas por el movimiento estudiantil de Córdoba.

De esta manera, la asistencia libre a las clases y la periodicidad de los exámenes no solo será un reclamo gremial de los estudiantes sino un principio que cuestiona el poder arbitrario de los profesores, y a la vez, que reconoce en el estudiante un sujeto activo que desafía la enseñanza tradicional y atrasada. Como señalará en el prólogo del libro "la asistencia libre - postulado reformista - vino a proclamar la bancarrota de la cátedra" que significaba la obediencia de asistir a escuchar a profesores que no reconocían como verdaderos maestros. Por su parte, el reclamo de periodicidad de los exámenes era una respuesta frente a control de los profesores que, al limitar la cantidad de exámenes, restringían la titulación de los estudiantes. La renovación de los métodos de enseñanza será otro de los postulados de la Reforma a través de seminarios de investigación de base experimental y la docencia libre como correlato de la asistencia libre que posibilita a los profesores ofrecer libremente seminarios o cursos con nuevas temáticas.

Lejos de abandonar el sentido político de La Reforma Universitaria, otros capítulos del mismo libro profundizarán en la importancia de la "extensión universitaria" como expresión del compromiso social de los estudiantes con las causas y luchas de los distintos sectores sociales, todos aspectos que configuran el "nuevo espíritu reformista" que adquiere el movimiento en su proyección continental.

Con todo, este libro contiene en germen la idea de un nuevo modelo de universidad que radicaliza los principios de la Reforma Universitaria y redefine la misma idea de "autonomía universitaria". Resumiendo, esta propuesta, González postulará no solo la necesidad de profundizar el carácter democratizador de la reforma sino también su propia

Universidad Nacional de Quilmas, 2008.

8 Para un análisis sobre las discusiones y posicionamientos políticos del Partido Socialista y del papel de González en dichos debates, véase: HERRERA, Carlos Miguel. Corrientes de izquierda en el socialismo argentino, 1932-1955. Nuevo Topo Revista de historia y pensamiento crítico, Prometeo, Buenos Aires n. 2, abr./may. 2006. 
superación hacia un modelo de universidad que, para cumplir su función crítica y de producción de ideas debe "emanciparse" del Estado, esto es, debe transformarse en una "Universidad Libre". Estas ideas serán el tema excluyente de su siguiente libro La emancipación de la universidad, contribución al estudio de un nuevo régimen superior en la Argentina que publicará en 1929, el cual constituye la base del proyecto de ley que presentará en 1941, siendo diputado nacional.

De esta manera, González retoma en prólogo aquellos señalamientos realizados en su libro anterior donde ubica la renovación que introduce la Reforma en línea de continuidad con "un proceso similar que se está operando en otros países regidos como el nuestro por el principio del monopolio de Estado en la cultura superior". (GONZÁLEZ, 1929, p. XVI). ¿Qué característica tiene este proceso? Para González, la "radical transformación" que atraviesan las instituciones de educación en Alemania, España e Italia y otros países, no es otra que la "desvinculación de la función burocrática de habilitación profesional y asignarles un rol puramente científico".

Esta tendencia que observa en la reforma Gentile de 1923 en Italia y en el proyecto de reforma elevada al parlamento español en 1928 tiene un común diagnóstico que reconoce no solo la incompatibilidad entre la función de habilitación profesional y la función científica de la universidad sino también la raíz de la decadencia de estas instituciones al transformarlas en una "fábrica de profesionales". Así, la causa de la burocratización de la universidad y la falta de espíritu científico y de fines culturales se debe a lo que denomina "profesionalismo", por ello - en un texto posterior - no dudará en señalar que "la universidad argentina padece un mal congénito que, como todas las de su género, la ha hecho fracasar hasta ahora: la función, por añadidura, exclusiva, de habilitación profesional". (GONZÁLEZ, 1945, p. 278).

González no será el único intelectual que criticará el carácter profesionalista de la universidad puesto que este argumento está presente en las intervenciones de su amigo y compañero Carlos Sánchez Viamonte, en las del pedagogo Ernesto Nelson, quién publicará en 1919 el libro Nuestros males universitarios, y aún más atrás en el tiempo en la contribución del intelectual católico José Manuel Estrada en los debates de la convención constituyente de la Provincia de Buenos Aires, en 1873. Todos ellos introducirán con distinto énfasis la necesidad de separar la habilitación profesional de la función científica de la universidad $^{10}$. Recuperando estos aportes, González formulará de la siguiente manera su propuesta para resolver la contradicción entre ambas funciones:

Por lo tanto se habría dado con la solución de tan grave problema, emancipando a la universidad del Estado, a lo que se llegaría -dando por resuelta la fase económica- con la institución del "examen de Estado", que significa la separación del título profesional y el título académico, dejando el primero a cargo directo del Estado y el segundo por cuenta exclusiva de la universidad. Para conquistar su independencia -no su autonomía- ella necesita, más que rentas propias, librarse de la obligación subalterna de fabricar profesionales, entregándose, como exponente de la sociedad y no como oficina del Estado, exclusivamente a la actividad científica, la elaboración de ideas y la cultural moral. (GONZÁLEZ, 1929, p. XXIII).

\footnotetext{
${ }^{9}$ La cita transcribe lo señalado en el "Prólogo" de GONZÁLEZ, Julio V. Reforma Universitaria. 2 volúmenes. Buenos Aires: Edición de la Revista Sagitario, 1927. p. 17.

10 Sobre el pensamiento universitario y educativo de Ernesto Nelson y José Manuel Estrada pueden consultarse los siguientes trabajos: Herrero (2006) y Torrendel (2008).
} 
Ciertamente, la propuesta del "examen de Estado" recupera la tradición universitaria de los países europeos y Norteamérica que precisamente deja en manos del Estado la regulación de las profesiones comúnmente llamadas "liberales". Pero el argumento de González va más allá de esta cuestión porque, en rigor, plantea una nueva y diferente relación entre el Estado y la Universidad. En este sentido, la idea de la "Universidad Libre" expresa una concepción radicalmente diferente al concepto de autonomía que finalmente primará en la Argentina y en varios países latinoamericanos, la cual está asociada a la problemática tensión entre su dependencia estatal y su reclamo de soberanía. Para González, la universidad debe conquista su independencia porque es una institución de la sociedad cuyos fines son la libre cultura y la ciencia, mientras siga bajo la tutela del Estado, este último impondrá sus funciones burocráticas.

En buena medida, el libro La emancipación de la universidad se plantea como un ensayo histórico que, tomando como foco la evolución de la Universidad de Buenos Aires, intenta demostrar el "doble movimiento simultáneo y paralelo: por una parte, de gradual desprendimiento del Estado y por la otra, de conversión hacia la sociedad". (GONZÁLEZ, 1929, p. XXIV). Este impulso hacia la emancipación - señala González - prácticamente está en los inicios de la universidad, aunque se materializará recién en 1871 con el proyecto de reorganización de Juan María Gutiérrez por entonces rector de la institución.

Fundada en 1821 en el período pos-independentista, la Universidad de Buenos Aires si bien se erigió a instancias del poder estatal, durante los años de gobierno del caudillo Juan Manuel de Rosas inicia su marcha hacia la emancipación como consecuencia de la pérdida de todo apoyo del poder político. Luego de la batalla de Caseros en 1852 que marca la caída de Rosas, la universidad vuelve a la tutela estatal, no obstante, con el proyecto de ley orgánica de Gutiérrez comenzará un largo debate que González resume de la siguiente forma:

La sociedad vindica frente al Estado el derecho inherente a su naturaleza de darse por sí misma su cultura. Para esto quiere la universidad libre y responsable solo ante aquella. La colectividad intenta sacudir la tutela del Estado porque es una traba en su libre desarrollo, coartado por la imposición de responder a los fines del Estado y de servir sus dogmas, y para obtenerlo procura la conquista de la universidad como uno de los instrumentos de su independencia. (GONZÁLEZ, 1929, p. 65).

El proyecto de Gutiérrez ciertamente proponía la independencia absoluta de la universidad que en la interpretación de González sería el caso de la "universidad libre, corporación privada u órgano de la sociedad", la cual generó resistencia por cuanto se señalaba críticamente la incompatibilidad de esa libertad con su dependencia económica de los poderes públicos. Nótese en la caracterización anterior que el sentido de "privado" que asigna González no se asimila a fines de lucro económico sino como diferente a lo estatal que remite a la esfera societal ${ }^{11}$.

Aunque no llegaría a tratarse en el congreso, el estatuto de la universidad sería incorporado en la Constitución de la Provincia de Buenos Aires de 1873 que sancionará la

\footnotetext{
11 "La teoría universitaria moderna no discute el derecho el Estado a controla la idoneidad para el ejercicio tanto de funciones públicas como de las profesiones liberales, lo que le niega es la facultad de hacer lo mismo en toda actividad del individuo que, como la de elaboración de cultura, está al margen de las necesidades e intereses del Estado. Y este es el caso de la universidad libre, corporación privada u órgano de la sociedad destinado a aquella función de individuos por medio del progreso de las ciencias y la formación de individuos hechos a las respectivas disciplinas mentales." (GONZÁLEZ, 1929, p. 72).
} 
separación de funciones entre el Estado y la universidad donde ésta última "expedirá exclusivamente grados académicos y el Estado exclusivamente títulos profesionales". (GONZÁLEZ, 1929, p. 84). De esta manera, la constitución provincial establecía la coexistencia de una universidad libre y otra oficial o del Estado, aunque en la práctica solamente existía una. No obstante, la falta de instrumentación de la universidad oficial encargada de los Exámenes de Estado y la posterior sanción de una ley que reglamenta el ejercicio de las profesiones liberales que asigna a la universidad aquella función que explícitamente había separado la constitución, contribuyeron a nuevamente superponer ambas funciones ${ }^{12}$.

Con la federalización de la ciudad de Buenos Aires en 1880, también aconteció la nacionalización de la Universidad de Buenos Aires que, según González, supuso un "cambio de amo", hecho que lentamente hará sentir su influencia en el Estatuto Provisorio de 1883 y en la posterior sanción de la Ley Avellaneda (Ley 1597) en 1885, primera normativa nacional que regulará las tres universidades existentes (Buenos Aires, Córdoba y La Plata). Al respecto, la historiografía de la universidad argentina ha consagrado la Ley Avellaneda como aquella normativa que estableció y legitimó el principio de la autonomía universitaria, a partir de cuatro artículos donde se define el gobierno, la organización institucional y la forma de designación de los profesores, y a su vez, delega en las universidades la tarea de dictar sus propios estatutos.

Quizás por la amplitud de este consenso, contrasta hoy aún más el análisis crítico de González que no duda en caracterizar esta normativa como una "ley contra su medio originario" ya que lejos de sancionar su emancipación de la universidad "vióse por la sanción de la Ley Avellaneda irremediablemente uncida al yugo burocrático, con la función que se le impuso de ejercer como oficina de Estado el monopolio de los títulos profesionales". (GONZÁLEZ, 1929, p. 123). Así, y pese a contar en el debate parlamentario con el conocimiento de las discusiones suscitadas en Bélgica y Francia sobre régimen universitario, la ley terminaría convalidando los errores anteriores cuando en su artículo 4 señala como obligación de la universidad de "expedir exclusivamente los diplomas de las respectivas profesiones científicas". Para González esta última denominación (profesiones científicas) constituye una "inconcebible confusión de conceptos" puesto que la ciencia y las profesiones son ordenes distintos que conllevan funciones diferentes, por ello es que considera un "agravio a la ciencia que importa enmaridarla con las profesiones". (ibidem, p. 124).

Lejos de profundizar el camino hacia la emancipación de la universidad, la Ley Avellaneda terminará no solo legitimando la función burocrática sino también profundizando la orientación profesionalista, raíz de los males universitarios. Las razones de este retroceso, González las encuentra, por un lado, en la falta de implementación de la universidad oficial encargada de los exámenes de Estado que llevó a persistir en el error de superponer ambas funciones en la misma institución, y por otro, en la tendencia

\footnotetext{
12 "Estos dos hechos sembraron de confusión, y al gravitar la realidad actuante por sobre los conceptos, resulto que había una sola universidad perteneciente al Estado que por la constitución otorgaba grados científicos y por la ley del 75 títulos profesionales, las cuales, emanando de un mismo organismo y por un mismo acto, resultaban en el hecho y automáticamente, patentes científico-profesionales, desde que uno y otro emanaban del Estado. Los grados científicos, al ser conferidos por una corporación científica y a la vez órgano del Estado, eran otorgados por aquella y colacionados por éste en un mismo acto." (GONZÁLEZ, 1929, p. 107).
} 
"utilitarista" de la sociedad argentina como producto del progreso económico que terminará transformando las instituciones universitarias en una "fábrica de profesionales"13. Resumiendo, esta caracterización señalará que:

No es aventurado afirmar que la era en la vida de la universidad inaugurada por la ley Avellaneda, se inicia con una marcada derivación de los estudios hacia el fin profesional. [...] La universidad comienza a atosigarse de profesionalismo. La flamante ley precipitó esa mala tendencia en la institución, cuando pudo detenerla. El fin que se le impuso significó la incorporación de la casa de altos estudios científicos, a la vida de un instituto politécnico. [...] La universidad no fue esto ni pudo serlo, porque la ley Avellaneda ya la había tocado de utilitarismo con su imposición del título profesional. (GONZÁLEZ, 1929, p. 149-150).

Ciertamente, la crítica al profesionalismo y la necesidad de emancipación de la universidad que postula González contrastará no solo con los sectores dominantes que procurarán mantener bajo control estatal a las casas de altos estudios sino también con los sectores medios que, como señala Juan Carlos Tedesco (1993, p. 173), adoptarán también el patrón tradicional que visualiza en las carreras profesionales (abogados, médicos e ingenieros) el camino hacia el ascenso social. Este posicionamiento de González también será la causa del carácter marginal de su posición dentro del Partido Socialista que expresión política de los sectores obreros y de la pequeña burguesía urbana realizarán una férrea defensa de las leyes educativas (Ley 1420 de Educación Común y Ley Avellaneda), sancionadas durante el período oligárquico liberal.

En buena medida, esta dinámica social que se consolidará en las primeras décadas del siglo XX explica el predominio de las demandas democratizadoras de la universidad, en términos de participación en el gobierno, que relegarán a un segundo plano una redefinición más profunda del modelo de universidad. Esto es lo que observa González en la posterior evolución de la Universidad de Buenos Aires que, si bien en la reforma de los estatutos de 1904 marca un primer paso en su democratización vuelve a consagrar "el funesto dogma de sus tres funciones simultáneas: científica, profesional y social' (ibidem, p. 198, grifos del autor) pese a que, en los debates previos, una vez más se alzaron voces contra la asimilación de la función científica y profesionalizante ${ }^{14}$.

Esta minuciosa reconstrucción histórica de la universidad que realiza González está en la base de su mirada crítica sobre la propia Reforma Universitaria de 1918 con la autoridad que le da haber sido uno de sus principales dirigentes estudiantiles. En rigor, su crítica no se dirige al contenido político que, por demás, él como intelectual siempre ubicó

\footnotetext{
13 "Hay que repetirlo ahora después de las reseñas demostrativas: la universidad de la ley Avellaneda organizada con un criterio empírico y definida en la orientación de sus estudios como positivista y utilitaria, por imposición del medio en que comenzaba a vivir, no pudo ser sino lo que fue: fábrica de profesionales." (GONZÁLEZ, 1929; p. 156, grifos míos). Y más adelante agrega: "La dependencia del Estado, la subordinación de las actividades a la expedición del título profesional, la tendencia inusitada hacia la labor científica chocando con la muralla burocrática, la plétora de profesionales, el utilitarismo manifestado en la forma de estudios prácticos o a lo sumo de técnica científica, el hibridismo de su carácter científicoprofesional, no eran sino diversos aspectos de un fenómeno: la universidad, con su fin propio de elaboración científica y función cultural, forzada por una mala ley a torcer su destino por la imposición de tareas correspondientes a una repartición del Estado." (GONZÁLEZ, 1929, p. 158).

14 "Si aquel de 1904 no tuvo la trascendencia que debió tener fue porque, desoyendo la advertencia del ministro Fernández, se trazaron estrechas y rígidas directivas, que obligaron a encauzar la caudalosa corriente de renovación dentro de los canales insuficientes de la ley Avellaneda, cuya intangibilidad fue dogma de los hombres de la universidad en aquella hora de su vida." (GONZÁLEZ, 1929, p. 204, grifos del autor).
} 
como expresión de una nueva generación cuya función es la transformar aquel orden en decadencia, sino a los límites del movimiento reformista que terminará ratificando el principio sancionado en la Ley Avellaneda de una universidad tutelada por el Estado. Así, refiriéndose a las resoluciones del primer congreso nacional de estudiantes universitarios reunidos en Córdoba señala que:

\begin{abstract}
Era, como se ve, repetir el funesto estribillo de la universidad esclavizada y comulgar una vez más con el dogma de la ley intangible. Estos enunciados, que merecieron la sanción unánime del congreso de estudiantes de Córdoba, fueron y los son hasta hoy, reconocidos de hecho en los diez años que lleva en actividad el movimiento reformista. Las reformas provocadas ese mismo año de 1918 en la Universidad de Córdoba, de 1919 y 1923 en la de Buenos Aires y de 1920 en la de La Plata, no sintieron el peso del yugo de la ley Avellaneda que somete a las universidades a la tutela del Estado. Bajo ese aspecto la Reforma de 1918 se mantuvo en los mismos límites de la de 1904. (GONZÁLEZ, 1929, p. 245, grifos míos).
\end{abstract}

Electo diputado nacional por el Partido Socialista en 1941, González presentará un proyecto de "Ley orgánica para la Universidad Nueva" que condensa más de veinte años de investigación histórica sobre la reforma y de reflexión sobre la universidad argentina. En los fundamentos del proyecto nuevamente realiza un balance del devenir de la universidad y el persistente problema de la función burocrática de la habilitación profesional que aleja de los fines específicos de la producción científica y cultural ${ }^{15}$, no obstante, el contexto socio-político y la propia realidad universitaria era otra, cuestiones que explican en parte las razones del porque dicho proyecto nunca sería tratado por el parlamento.

\title{
A modo de conclusiones
}

Como señalamos en un trabajo anterior, la recurrente inestabilidad institucional que atravesó las universidades durante el siglo XX producto de los golpes militares de 1930, 1943, 1955, 1966 y 1976 marcó fuertemente no solo la relación entre la Universidad y el Estado sino también moldeó el vínculo entre intelectuales universitarios y política (SUASNÁBAR, 2009). De tal manera, las constantes intervenciones a la universidad fueron sedimentando un comportamiento refractario hacia cualquier intromisión estatal en la vida de las universidades, el cual terminó configurando un sentido negativo de autonomía entendido como no intervención. Por su parte, la experiencia de la Reforma no solo generó una identidad universitaria sino también instituyó una modalidad de intervención política expresada en la participación de los intelectuales en los debates públicos manteniendo su autonomía respecto de los partidos políticos, situación que se profundizará también por el recelo de estos últimos hacia los intelectuales. En este sentido, la convergencia entre ambos procesos explica en parte la concepción aún dominante en amplias franjas intelectuales que asocia la defensa de la autonomía con la reforma universitaria. La Ley Avellaneda parecería señalar el punto de articulación que como analizamos será legitimada por la Reforma Universitaria.

La recuperación del pensamiento de Julio V. González, dirigente estudiantil y uno de los creadores del ideario reformista nos revela una concepción radicalmente diferente de la idea de autonomía universitaria que estuvo (y aún sigue estando) olvidada en los debates universitarios. Dicha ausencia se explica por el carácter revulsivo de la reflexión

${ }^{15}$ Sobre el proyecto de González, véase el trabajo de Bonaldi (1995).

Hist. Educ. (Online)

Porto Alegre

v. 22

ก. 54

jan./abr. 2018

p. $174-189$ 
gonzaliana que en los orígenes de nuestras instituciones postulaba no solamente la necesidad de profundizar el carácter democratizador de la reforma sino también su propia superación hacia un modelo de universidad que para cumplir su función crítica y de producción de ideas debe "emanciparse" del Estado.

Al respecto, vale recordar una vez más que el nudo problemático que plantea González es el profesionalismo que genera la función burocrática que el Estado transfiere a las universidades. En este marco se inscribe la actualidad de discutir un nuevo modelo de universidad que como señalaba González no puede transformarse en una mera fábrica de profesionales sino también productora de conocimiento científico y pensamiento crítico.

Por último, la figura de Julio V. González también nos permite reflexionar sobre la siempre compleja relación entre intelectuales y política que en su derrotero nos revela aquel proceso de radicalización como un movimiento de autoconciencia (revuelta estudiantil, joven generación, socialista humanista), y a la vez, de cambio en la modalidades de intervención (de "intelectual comprometido" al "intelectual orgánico") pero que conservará una mirada autónoma y crítica aún de las posiciones partidarias.

\section{Fuentes primarias}

GONZÁLEZ, Julio V. EI Partido Nacional Reformista. In: CIRIA, Alberto; SANGUINETTI, Horacio. Los reformistas. Buenos Aires: Jorge Álvarez, 1968.

La emancipación de la universidad. Contribución al estudio de un nuevo régimen superior en la Argentina. Buenos Aires: Edición de los Talleres Gráficos Argentinos, 1929. 1927.

La reforma universitaria. 2 vols. Buenos Aires: Edición de la Revista Sagitario,

La revolución universitaria 1918-1919. Buenos Aires: Cooperativa editorial Nosotros, 1922.

. La Universidad. Teoría y acción de La Reforma. Buenos Aires: Claridad, 1945. 1931.

Reflexiones de un argentino de la nueva generación. Buenos Aires: Claridad,

\section{Referencias}

BONALDI, Pablo. Proyecto de ley de Julio V. González. Revista Pensamiento Universitario, n. 3, año 3, p. 66-70, 1995.

GRACIANO, Osvaldo. Entre la torre de marfil y el compromiso político. Intelectuales de izquierda en la Argentina 1918-1955. Buenos Aires: Universidad Nacional de Quilmas, 2008.

HERRERA, Carlos. Corrientes de izquierda en el socialismo argentino, 1932-1955. Nuevo Topo Revista de historia y pensamiento crítico, Buenos Aires, Prometeo, n. 2, p. 4249, abr./may. 2006.

Generación y política. In: GONZÁLEZ, Julio V. Revista Socialista, Cuarta Época, Buenos Aires, Año I, n. 1, jun. 2009. p. 70-82.

HERRERO, Abel. Liberalismo y democracia en Argentina. El estudio de un caso: Ernesto Nelson: ¿un educador del Estado en contra del Estado? Revista Utopía y Praxis

Latinoamericana, Universidad de Zulia, Maracaibo, Venezuela, v. 11, n. 33, p. 103-108, 
abr.jjun. 2006.

KOHAN, Néstor. De Ingenieros al Che. Ensayos sobre el marxismo argentino y latinoamericano. Buenos Aires: Ed. Biblos, 2000.

MÚNERA RUIZ, Leopoldo. La Reforma de Córdoba y el gobierno de las universidades públicas en América Latina. Análisis comparado de cinco universidades. Revista Ciencia Política, v. 6, n. 12, p. 6-40, 2011.

SUASNÁBAR, Claudio. La Reforma Universitaria del 1918: una mirada histórica de la relación entre intelectuales, universidad y política en la Argentina. Revista Práxis

Educativa, Ponta Grossa, v. 4, n. 1, p. 51-61, jan./jun. 2009.

TEDESCO, Juan Carlos. Oligarquía, clases media y educación en Argentina (1900-1930), Educación y Sociedad en la Argentina (1880-1945). Buenos Aires: Ed. del Solar, 1993. p. 173-214.

TORRENDEL, Carlos. A destiempo: José Manuel Estrada y la autonomía universitaria. In: Jornadas de Historia de la Educación, XV, 2008, Salta. Anales... Salta: SAHE-UNSa, 2008, p. 151-159.

TUNNERMAN BERNHEIM, Carlos. La Reforma de Córdoba. Vientre fecundo de la transformación universitaria. In: SADER, E.; ABOITES, H.; GENTILI, P. La Reforma Universitaria. Desafíos y perspectivas noventa años después. Buenos Aires: CLACSO, 2008. p. 15-19.

CLAUDIO SUASNÁBAR es Doctor en Ciencias Sociales (Flacso), Universidad Nacional de La Plata (UNLP), Argentina, Instituto de Investigaciones en Humanidades y Ciencias Sociales (IdIHCS) - Consejo Nacional de Investigaciones Científicas y Tecnológicas (Conicet). Endereço: Calle 45, 889 PB, Dpto. F entre 12 y 13 - La Plata - Buenos Aires - Argentina. E-mail: csuasnabar@gmail.com

Recebido em 07 de novembro de 2017. Aceito em 04 de dezembro de 2017. 